\title{
CONFÉRENCE
}

\section{Expression orale des maladies bulleuses auto-immunes.}

\section{Pr Joly P}

Centre de référence des maladies bulleuses auto-immunes, Clinique Dermatologique - CHU Rouen

Les maladies bulleuses auto-immunes (MBAI) comprennent plusieurs maladies liées à la production d'autoanticorps pathogènes responsables de la formation de bulles au sein de l'épiderme. On distingue le pemphigus, dans lequel les auto-anticorps ciblent les protéines d'adhésion interkératinocytaires (protéines desmosomales) et les dermatoses bulleuse de la jonction dermo épidermique (pemphigoïde bulleuse, pemphigoïde cicatricielle, pemphigoïde gestationis, dermatose à lgA linéaire, épidermolyse bulleuse acquise) dans lesquelles les auto-anticorps se fixent sur différentes protéines de la jonction épiderme-derme (protéines hémi-desmosomales, de la lamina lucida, lamina densa, ou des fibres d'ancrage du derme superficiel). Chacune de ses maladies peut comporter des lésions bucales, correspondant habituellement à des érosions post bulleuses d'évolution trainante, entrainant douleur et dyspahagie et pouvant résulter en une perte de poids t parfois importante. Le pemphigus vulgaire et la pemphigoide cicatricielle se présentent habituellement avec des lésions buccales isolées. Le diagnostic est souvent fait très tardivement (3 à 9 mois en moyenne), ce qui est à l'origine d'un ressentiment durable des patients contre les différents médecins n'ayant pas fait le diagnostic. Les principaux diagnostics différentiels sont les aphtes, la stomatite bulleuse hémorragique, et le lichen plan érosif qui ne comporte pas toujours les lésions blanchâtres leucoplasiques caractéristiques.

Depuis 30 ans, la recherche clinique et biologique sur les MBAI a considérablement avancé et permis l'identification et le clonage des antigènes cibles et la compréhension des mécanismes lésionnels. Le diagnostic de ces maladies rares se fait sur l'aspect clinique des lésions, l'histologie cutanée et l'identification et le dosage des anticorps anti-épiderme dans le serum. La biopsie pour examen histologique doit être réalisée soit sur une lésion bulleuse si elle est présente, soit à la jonction zone érosive zone saine mais jamais en zone érosive. La biopsie pour examen en immunofluorescence directe (IFD) doit être réalisée sur une zone de muqueuse péri-lésionnelle NON érosive. L'IFD reste le gold standard en matière de diagnostic des MBAl. Elle montre un marquage en résille au sein de l'épiderme dans le pemphigus, et un marquage linéaire de la jonction dermo épidermique dans les dermatoses bulleuses de la jonction. La réalisation d'une IFD en peau séparée par le Nacl molaire permet de distinguer les dermatoses jonctionnelles superficielles (essentiellement la pemphigoïde bulleuse) dans lesquelles le marquage concerne le toit de la zone de clivage, des dermatoses profondes, dans lesquelles le marquage concerne le plancher de la zone de clivage. Les examens sérologiques aident pour discriminer les différentes dermatoses jonctionnelles. Ils ont également un intérêt dans le suivi pour mesurer l'activité de la maladie au cours du pemphigus et de la pemphigoïde bulleuse.

Pascal.Joly@chu-rouen.fr

(C) The authors, published by EDP Sciences. This is an Open Access article distributed under the terms of the Creative Commons Attribution License 4.0 (http://creativecommons.org/licenses/by/4.0/). 